\section{A Description of Hiker Preparedness in New Hampshire}

Background.-Outdoor recreation, specifically hiking, has increased dramatically over the last 30 years. While many enjoy their hikes without incident, others find themselves caught unprepared and in need of rescue. Mobilization of gear, vehicles, and personnel incur risk and a burden on local resources. Education initiatives focusing on hiker preparedness have been developed in an effort to prevent injuries and rescues. However, their effects on hiker habits have gone unexamined.

Objective.-The study objective was to describe hiker habits in the categories of demographics, gear, planning, and communication devices. Our hypotheses were that most hikers did not carry all 10 essential items (deemed by New Hampshire's "Hike Safe" Initiative) and that carrying a communication device (cell/smartphone, GPS) encourages hikers to attempt more difficult trails.

Methods.-A cross-sectional, convenience survey was conducted at 3 trailheads in the White Mountain National Forest of New Hampshire during weekends in summer 2011. The study group consisted of hikers who consented to an IRB-approved questionnaire before their trip.

Results.-A total of 199 hikers completed surveys. Of the respondents, $59.8 \%$ were male and $40.2 \%$ female $(n=195)$, 20-29 years old was the most common age group at $29.2 \%(\mathrm{n}=$ $195)$, with $58.1 \%(\mathrm{n}=191)$ planning a hike between 4 and 12 hours. Only $17.8 \%(\mathrm{n}=197)$ of hikers carried all 10 items, the least being whistles at $43.1 \%$ and compasses at $46.2 \%(\mathrm{n}=$ 197). Of the hikers, $90.3 \%(\mathrm{n}=196)$ carried a communication device and $71.8 \%(n=156)$ were "likely" to choose the same hike without it.

Conclusions.-Most hikers do not carry the 10 essential items and do not choose more difficult trails because of their communications devices. Future education efforts should focus on the importance of compasses and whistles. The small sample size and single study location prevent this study from giving a generalized description of hiking habits.

Ryan Mason

Kenneth Williams, MD

Selim Suner, MD, MS

Providence, $R I$

\section{Long-Term Neurological Sequelae After Latrodectus geometricus (Brown Widow Spider) Envenomation: A Case Report}

Latrodectus geometricus, also known as the brown widow spider, is one of 5 United States species from the widow family. Its venom, characterized as a neurotoxin, produces symptoms including widespread muscle spasm. The toxin has been known to mimic severe abdominal disorders such as acute appendicitis and ruptured ovarian cysts. We report a single case involving a previously healthy adult male who presented to the emergency department with neuropathy and foot drop after confirmed envenomation with the neurotoxin from a brown widow spider (Latrodectus geometricus). His injury occurred approximately 2 weeks prior to presentation. MRI of the lumbar spine revealed an enhanced paraspinal muscular lesion at T2 extending into the gluteal muscles. Cerebrospinal fluid analysis was normal. EMG and nerve conduction studies were compatible with peripheral neuropathy, active denervation of the right gastrocnemius muscle, and signs consistent with active demyelination. Neurologic manifestation of typical radiculopathy was clouded by paraspinal muscular inflammation related to rhabdomyolysis. He was eventually diagnosed with mononeuritis, right foot drop, moderate rhabdomyolysis, and severe neuropathic pain. His severe neurological devastation was attributed to envenomation with the brown widow spider neurotoxin. His symptoms persisted for more than 7 months after initial exposure and led to significant alterations in the patient's quality of life. A plethora of information exists explaining the short-term complications of widow spider envenomation. Very little literature exists concerning long-term neurological complications of widow toxin exposure, particularly brown widow spider envenomation. Awareness of the possibility of devastating complications is beneficial and will aid in future development and institution of early preventive management.

Ogadinma I. Obie, MD Elizabeth Phillips, MD Andrew Edwards, MD Birmingham, $A L$

\section{Changes in Peripheral and Cerebral Oxygenation With Altitude and Acclimatization}

Background.-Previous studies have addressed the changes of peripheral or cerebral oxygenation that occur with ascent to altitude and acclimatization. None of these studies have explicitly examined the relationship between peripheral and cerebral oxygenation.

Methods.-11 subjects from the Xtreme Alps medical research expedition were studied at sea level and 2 and 7 days after ascent to $4559 \mathrm{~m}$. Near-infrared spectroscopy and pulse oximetry were used to measure cerebral and peripheral oxygenation, respectively, while capnography was used to measure end-tidal $\mathrm{CO}_{2}$.

Results.-Peripheral oxygenation decreased from $98 \% \pm$ $1.4 \%$ at sea level to $80.2 \% \pm 4.7 \%$ at day 2 at altitude before rising to $85.1 \% \pm 4.7 \%$ after 7 days. While peripheral oxygenation dropped initially before rebounding at altitude, cerebral oxygenation continued to decline, decreasing from $68.8 \% \pm 4.0 \%$ at sea level to $65.0 \% \pm 6.2 \%$ after 2 days at altitude and further to $62.3 \% \pm 4.7 \%$ at day 7 . End-tidal $\mathrm{CO}_{2}$ decreased from $4.5 \pm 0.8 \mathrm{kPa}$ at sea level to $3.4 \pm 0.4 \mathrm{kPa}$ after 2 days at altitude and to $3.1 \pm 0.5 \mathrm{kPa}$ after 7 days at altitude. Both resting $\mathrm{SpO}_{2}(\rho=.441, P=.019)$ and $\operatorname{ETCO}_{2}(\rho=$ $0.491, P<.01)$ were correlated with cerebral oxygenation.

Conclusions.-Improvement in peripheral oxygenation is not a reliable predictor of increasing cerebral oxygenation at altitude, as this divergence in $\mathrm{SpO}_{2}$ and $\mathrm{SctO}_{2}$ between days 2 and 7 at altitude illustrates. This divergence may be attributable to the differences in the response to $\mathrm{CO}_{2}$ between the periphery and the central nervous system. At simulated extreme altitudes 
there is a significant correlation between subjects with a more pronounced hypoxic ventilatory response (HVR) and increased impairment on a variety of cognitive tasks. This contrasts with other reports demonstrating improved climbing performance in subjects with a higher HVR. This dichotomy between athletic and cognitive performance parallels the divergence between peripheral and cerebral oxygenation seen in the present study.

\author{
Matthew R. Sanborn, MD \\ Philadelphia, PA \\ Mark E. Edsell, MBChB \\ Heng Yow, MBChB \\ Daniel S. Martin, MBChB \\ London, $U K$ \\ Chris Imray, MBBS \\ Coventry, $U K$ \\ Mike Grocott, MBBS \\ Southampton, UK
}

\section{Cerebral Hemodynamics at Altitude Using Diffuse Correlation Spectroscopy and Transcranial Doppler}

Background.-Studies of cerebral hemodynamics at altitude have been hampered by the lack of a portable, real-time, noninvasive monitor of intracranial blood flow. Most studies to date have relied on cerebral blood flow velocities (CBF-V) measured by transcranial Doppler, as a surrogate for cerebral blood flow. The use of this measurement to accurately reflect cerebral blood flow is predicated on the recently disproven notion that middle cerebral artery (MCA) diameter remains constant with changes in altitude.

Methods.-We used a novel technology, diffuse-correlation spectroscopy (DCS), which is not dependent on MCA diameter changes and has been previously validated against Xenon CT, to assess the cerebrovascular response to hyperventilation during acclimatization and compared this to traditional transcranial Doppler (TCD). Eleven subjects from the Xtreme Alps medical research expedition were studied at sea level and 2 and 7 days after ascent to $4559 \mathrm{~m}$ before and after hyperventilation to reduce end-tidal $\mathrm{CO}_{2}$ by $50 \%$. Transcranial Doppler was used to measure peak systolic velocity (PSV) in the MCA. Diffusecorrelation spectroscopy was used to measure changes in relative $\mathrm{CBF}(\mathrm{rCBF})$.

Results.-Diffuse-correlation spectroscopy revealed a decrease in $\mathrm{rCBF}$ of $19.6 \% \pm 18.9 \%$ following hyperventilation at sea level, $28.9 \% \pm 13.6 \%$ following hyperventilation after 2 days at altitude and $33.0 \% \pm 29.0 \%$ following 7 days at altitude. PSV as measured by TCD demonstrated a decrease of $32 \% \pm 12.7 \%, 28.9 \% \pm 32.3 \%$ and $-29.4 \% \pm 7.6 \%$ respectively.

Conclusions.-Diffuse-correlation spectroscopy provides a safe, portable, and noninvasive measure of cerebral blood flow at altitude, reflecting cerebral perfusion at the parenchymal level. By contrast TCD provides a measure of velocity of flow through large resistance vessels, with no direct information about end-organ perfusion. While DCS correlates poorly with
TCD, further studies are necessary to elucidate which test is a more accurate reflection of cerebral blood flow.

Matthew R. Sanborn, MD Meeri N. Kim, MA

Rickson Mesquita, PhD Arjun Yodh, $\mathrm{PhD}$ Philadelphia, PA

Mark E. Edsell, MBChB

Heng Yow, MBChB

Daniel S. Martin, MBChB

London, UK

Chris Imray, MBBS

Coventry, $U K$

Mike Grocott, MBBS

Southampton, UK

\section{Knowledge of Alcohol Impairment in Boaters}

Background.- Alcohol is a factor in at least $60 \%$ of boating-related fatalities. Prior literature has shown that $30 \%-$ $40 \%$ of the participants drank alcohol while boating, and they seldom knew the laws or dangers associated with alcohol ingestion while boating. To our knowledge, this is the first study to directly approach boaters at the dock to assess participants' knowledge regarding alcohol impairment while boating.

Methods.-This was a cross-sectional survey of a convenience sample of boaters aged 21 and older at 4 lakes in Illinois during July 2011. Participants were asked to fill out an 8-question survey covering knowledge about alcohol use and boating. Chi-square analysis was used to assess knowledge differences by demographic variables, as well as boat ownership and seating position. Kruskal-Wallis assessed differences by education level.

Results.-210 surveys were analyzed. The majority of participants correctly answered 4 of the 5 knowledge questions, including $84 \%$ correctly reporting the watercraft blood alcohol legal limit. Seventy-six percent admitted drinking alcohol while boating; $80 \%$ erroneously believed that it was more dangerous for the driver to be intoxicated than the passenger $(n=194)$. There were no differences in knowledge by gender, education, boat ownership, or seating position. Participants older than 40 years of age were more likely to know that being intoxicated makes one 10 times more likely to drown $(P<.05)$. Younger participants (age 21-40) were significantly more likely to report drinking while boating compared to older participants $(P<.05)$. Older participants were also more likely to own a boat and be drivers $(P<.05)$.

Conclusions.-A majority of participants imbibe while boating despite a basic understanding of the dangers in doing so. Public health officials may benefit from focusing education on the younger age group of boaters to help decrease alcoholrelated morbidity and mortality. 